Supplement of

\title{
Technical note: First comparison of wind observations from ESA's satellite mission Aeolus and ground-based radar wind profiler network of China
}

Jianping Guo et al.

Correspondence to: Boming Liu (liuboming@whu.edu.cn)

The copyright of individual parts of the supplement might differ from the CC BY 4.0 License. 

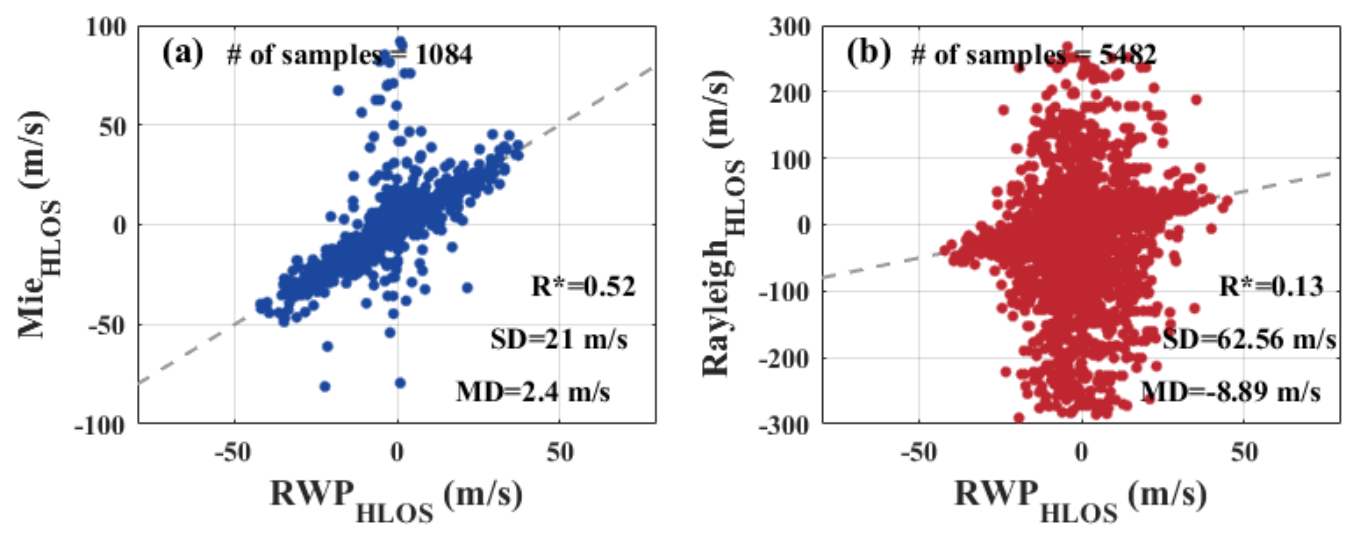

Figure S1. Aeolus against RWP HLOS winds for (a) Mie-cloudy winds and (d) Rayleigh-clear winds for all data. 

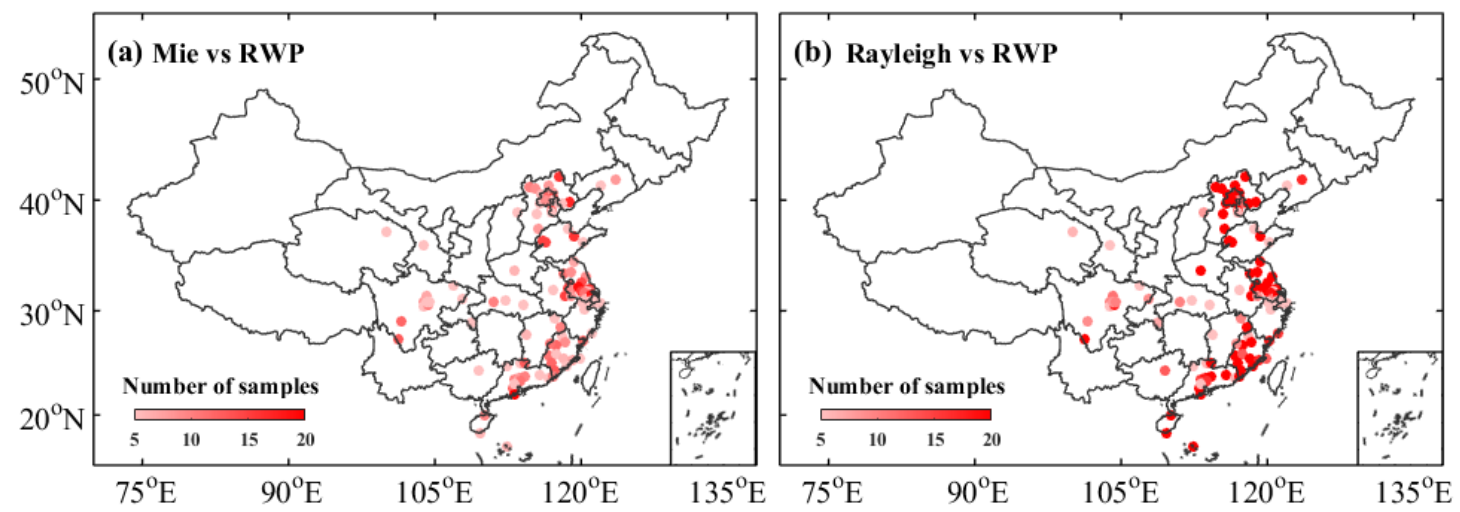

Figure S2. Spatial distribution of the number of paired data samples between Aeolus HLOS and RWP HLOS wind speeds at each RWP site. The wind measurements are separated in (a) Mie-cloudy winds and (b) Rayleigh-clear winds. 